Paru à Comptes Rendus de l'Académie des Sciences de Paris, Ser. I 347 (2009) 785-790.

\title{
INTERPOLATION AVEC CONTRAINTES SUR DES ENSEMBLES FINIS DU DISQUE
}

\author{
RACHID ZAROUF
}

\section{Résumé}

Etant donné un ensemble fini $\sigma$ du disque unité $\mathbb{D}=\{z \in \mathbb{C}:|z|<1\}$ et une fonction $f$ holomorphe dans $\mathbb{D}$ appartenant 'à une certaine classe $X$, on cherche $g$ dans une autre classe $Y$ (plus petite que $X$ ) qui minimise la norme de $g$ dans $Y$ parmi toutes les fonctions $g$ satisfaisant la condition $g_{\mid \sigma}=f_{\mid \sigma}$. On montre que dans le cas $Y=H^{\infty}$, la constante d'interpolation correspondante $c\left(\sigma, X, H^{\infty}\right)$ est majorée par $a \varphi_{X}\left(1-\frac{1-r}{n}\right)$ où $n=\# \sigma, r=\max _{\lambda \in \sigma}|\lambda|$ et $\varphi_{X}(t)$ est la norme de la fonctionnelle d'évaluation $f \mapsto f(t)$, sur l'espace $X$. La majoration est exacte sur l'ensemble des $\sigma$ avec $n$ et $r$ donné.

\begin{abstract}
Given a finite set $\sigma$ of the unit disc $\mathbb{D}=\{z \in \mathbb{C}:,|z|<1\}$ and a holomorphic function $f$ in $\mathbb{D}$ which belongs to a class $X$, we are looking for a function $g$ in another class $Y$ (smaller than $X$ ) which minimizes the norm $\|g\|_{Y}$ among all functions $g$ such that $g_{\mid \sigma}=f_{\mid \sigma}$. For $Y=H^{\infty}$, and for the corresponding interpolation constant $c\left(\sigma, X, H^{\infty}\right)$, we show that $c\left(\sigma, X, H^{\infty}\right) \leq a \varphi_{X}\left(1-\frac{1-r}{n}\right)$ where $n=\# \sigma, r=\max _{\lambda \in \sigma}|\lambda|$ and where $\varphi_{X}(t)$ stands for the norm of the evaluation functional $f \mapsto f(t)$ on the space $X$. The upper bound is sharp over sets $\sigma$ with given $n$ and $r$.
\end{abstract}

\section{Abridged English version}

The problem considered is the following: given $X$ and $Y$ two Banach spaces of holomorphic functions on the unit disc $\mathbb{D}=\{z \in \mathbb{C}:|z|<1\}, X \supset Y$, and a finite set $\sigma \subset \mathbb{D}$, to find the least norm interpolation by functions of the space $Y$ for the traces $f_{\mid \sigma}$ of functions of the space $X$, in the worst case of $f$.

The classical interpolation problems- those of Nevanlinna-Pick and Carathéodory-Schur (on the one hand) and Carleson's free interpolation (on the other hand)- are of this nature. Two first are "individual", in the sens that one looks simply to compute the norms $\|f\|_{H_{\mid \sigma}^{\infty}}$ or $\|f\|_{H^{\infty} / z^{n} H^{\infty}}$ for a given $f$, whereas the third one is to compare the norms $\|a\|_{l^{\infty}(\sigma)}=\max _{\lambda \in \sigma}\left|a_{\lambda}\right|$ and

$$
\inf \left(\|g\|_{\infty}: g(\lambda)=a_{\lambda}, \lambda \in \sigma\right) .
$$

Here and everywhere below, $H^{\infty}$ stands for the space (algebra) of bounded holomorphic functions in the unit disc $\mathbb{D}$ endowed with the norm $\|f\|_{\infty}=\sup _{z \in \mathbb{D}}|f(z)|$. Looking at this comparison problem, say, in the form of computing/estimating the interpolation constant

$$
c(\sigma, X, Y)=\sup _{f \in X,\|f\|_{X} \leq 1} \inf \left\{\|g\|_{Y}: g_{\mid \sigma}=f_{\mid \sigma}\right\},
$$

which is nothing but the norm of the embedding operator $\left(X_{\mid \sigma},\|\cdot\|_{X_{\mid \sigma}}\right) \rightarrow\left(Y_{\mid \sigma},\|\cdot\|_{Y_{\mid \sigma}}\right)$, one can think, of course, on passing (after) to the limit- in the case of an infinite sequence $\left\{\lambda_{j}\right\}$ and its finite sections $\left\{\lambda_{j}\right\}_{j=1^{-}}^{n}$ in order to obtain a Carleson type interpolation theorem $X_{\mid \sigma}=Y_{\mid \sigma}$. But not necessarily. In particular, even the classical Pick-Nevanlinna theorem (giving a necessary and sufficient condition on a function $a$ for the existence of $f \in H^{\infty}$ such that $\|f\|_{\infty} \leq 1$ and $f(\lambda)=a_{\lambda}, \lambda \in \sigma$ ), does not lead immediately to Carleson's criterion for $H_{\mid \sigma}^{\infty}=l^{\infty}(\sigma)$. (Finally, a direct deduction of Carleson's theorem from Pick's result was done by P. Koosis [10] in 1999 only). Similarly, the problem stated for $c(\sigma, X, Y)$ is of interest in its own. For this paper, the following question was especially stimulating (which is a part of a more complicated question arising in an applied situation in [2] and [3]): given a set $\sigma \subset \mathbb{D}$, how to estimate $c\left(\sigma, H^{2}, H^{\infty}\right)$ in terms of $n=\operatorname{card}(\sigma)$ and $\max _{\lambda \in \sigma}|\lambda|=r$ only? ( $H^{2}$ being the standard Hardy space of the disc).

Here, we consider the case of $H^{\infty}$ interpolation $\left(Y=H^{\infty}\right)$ and the following scales of Banach spaces $X$ :

(a) $X=H^{p}=H^{p}(\mathbb{D}), 1 \leq p \leq \infty$, the standard Hardy spaces on the disc $\mathbb{D}$, 
(b) $X=l_{a}^{2}\left(\frac{1}{(k+1)^{\alpha-1}}\right), \alpha \geq 1$, the weighted spaces of all $f(z)=\sum_{k \geq 0} \hat{f}(k) z^{k}$ satisfying

$$
\sum_{k \geq 0}|\hat{f}(k)|^{2} \frac{1}{(k+1)^{2(\alpha-1)}}<\infty .
$$

An equivalent description of this scale of spaces is: that

$X=L_{a}^{2}\left(\left(1-|z|^{2}\right)^{\beta} d x d y\right), \beta=2 \alpha-3>-1$, the Bergman weighted spaces of holomorphic functions such

$$
\int_{\mathbb{D}}|f(z)|^{2}\left(1-|z|^{2}\right)^{\beta} d A<\infty
$$

For the case $\beta=0$, we shorten the notation to $X=L_{a}^{2}$. For these two series of spaces we show

$$
c_{1} \varphi_{X}\left(1-\frac{1-r}{n}\right) \leq \sup \left\{c\left(\sigma, X, H^{\infty}\right): \# \sigma \leq n,|\lambda| \leq r, \lambda \in \sigma\right\} \leq c_{2} \varphi_{X}\left(1-\frac{1-r}{n}\right),
$$

where $\varphi_{X}(t), 0 \leq t<1$ stands for the norm of the evaluation functional $f \mapsto f(t)$ on the space $X$.

In order to prove the right hand side inequality, we first use a linear interpolation:

$$
f \mapsto \sum_{k=1}^{n}\left\langle f, e_{k}\right\rangle e_{k},
$$

where $\langle.,$.$\rangle means the Cauchy sesquilinear form \langle h, g\rangle=\sum_{k \geq 0} \hat{h}(k) \overline{\hat{g}(k)}$, and $\left(e_{k}\right)_{k=1}^{n}$ is the explicitly known Malmquist basis of the space $K_{B}=H^{2} \Theta B H^{2}, B=\prod_{i=1}^{n} b_{\lambda_{i}}$ being the corresponding Blaschke product, $b_{\lambda}=\frac{\lambda-z}{1-\bar{\lambda} z}($ see N. Nikolski, [12] p. 117)). Next, we use the complex interpolation between Banach spaces, (see H. Triebel [14] Theorem 1.9.3 p.59). Among the technical tools used in order to find an upper bound for $\left\|\sum_{k=1}^{n}\left\langle f, e_{k}\right\rangle e_{k}\right\|_{\infty}$ (in terms of $\|f\|_{X}$ ), the most important is a Bernstein-type inequality $\left\|f^{\prime}\right\|_{p} \leq c_{p}\left\|B^{\prime}\right\|_{\infty}\|f\|_{p}$ for a (rational) function $f$ in the star-invariant subspace $H^{p} \cap B \bar{H}_{0}^{p}$ generated by a (finite) Blaschke product $B$, (K. Dyakonov [7]). For $p=2$, we give an alternative proof of the Bernstein-type estimate we need.

The lower bound problem is treated by using the "worst" interpolation $n$-tuple $\sigma=\sigma_{\lambda, n}=\{\lambda, \ldots, \lambda\}$, a one-point set of multiplicity $n$ (the Carathéodory-Schur type interpolation). The "worst" interpolation data comes from the Dirichlet kernels $\sum_{k=0}^{n-1} z^{k}$ transplanted from the origin to $\lambda$. We notice that spaces $X$ of (a) and (b) satisfy the condition $X \circ b_{\lambda} \subset X$ but this is not the case for spaces $X$ described in (c) below for $p \neq 2$, which makes the problem of upper/lower bound more difficult.

Other spaces considered are the following:

(c) $X=l_{a}^{p}\left(\frac{1}{(k+1)^{\alpha-1}}\right), \alpha \geq 1,1 \leq p \leq \infty ;(\mathrm{d}) X=L_{a}^{p}\left(\left(1-|z|^{2}\right)^{\beta} d A\right), \beta>-1,1 \leq p \leq 2$.

For these spaces we also found upper and lower bounds for $c\left(\sigma, X, H^{\infty}\right)$ (sometimes for special sets $\sigma$ ) but with some gaps between these bounds.

\section{A. Introduction}

Le problème extremal d'interpolation est le suivant: étant donnés $\lambda_{1}, \ldots, \lambda_{n}$ dans $\mathbb{D}=\{z \in \mathbb{C}:,|z|<1\}, B=\Pi_{i=1}^{n} b_{\lambda_{i}}$ où $b_{\lambda}=\frac{\lambda-z}{1-\bar{\lambda} z}$, et $f \in \mathcal{H}$ ol $(\mathbb{D})$, on cherche à calculer ou estimer

$$
\|f\|_{H^{\infty} / B H^{\infty}}=\inf \left\{\|g\|_{\infty}: f-g \in B \mathcal{H o l}(\mathbb{D})\right\}
$$

Les problèmes classiques de Nevanlinna-Pick (1916) et de Carathéodory-Schur (1908), (voir [8] et [12] pour ces deux problèmes classiques et pour des références originales), en sont des cas particuliers correspondant respectivement à celui où les $\lambda_{j}$ sont $n$ points distincts et au cas où $\lambda_{1}=\lambda_{2}=\ldots=\lambda_{n}=0$.

\section{(1) Le sujet de cette note est une version de ce dernier problème:}


Trouver ou majorer/minorer la norme de la meilleure interpolante $\|f\|_{H^{\infty} / B H^{\infty}}$ en fonction de la taille de $f$ mesurée dans un espace de Banach $X$ de fonctions holomorphes dans $\mathbb{D}$, c'est à dire $\|f\|_{X}$. De façon plus précise, il s'agit de calculer ou majorer/minorer les constantes

$$
\begin{gathered}
c(\sigma, X, Y)=\sup _{f \in X,\|f\|_{X} \leq 1} \text { inf }\left\{\|g\|_{Y}: g_{\mid \sigma}=f_{\mid \sigma}\right\}, \text { et } \\
C_{n, r}(X, Y)=\sup \left\{c(\sigma, X, Y): \# \sigma \leq n, \forall j=1 . . n,\left|\lambda_{j}\right| \leq r\right\},
\end{gathered}
$$

lorsque $Y=H^{\infty}, r \in[0,1[$ et $n \geq 1$. Nous pouvons néanmoins faire quelques commentaires sur la constante $c(\sigma, X, Y)$ lorsque $Y$ est, comme $X$, un espace de Banach de fonctions holomorphes (inclus dans $X$ ).

\section{(2) Motivations pour ce problème}

(a) Le point de départ est une partie d'une question posée par Laurent Baratchart (communication orale) et provenant d'un problème d'approximation appliquée (voir [2] et [3]): trouver une estimation de $c\left(\sigma, H^{2}, H^{\infty}\right)$ en fonction de $\operatorname{card}(\sigma)=\operatorname{deg}(B)$ et de $\max _{\lambda \in \sigma}|\lambda|=r$.

Parmi d'autres résultats, voici ci-dessous la réponse obtenue:

$$
\left(\frac{\frac{1}{32} n}{1-r}\right)^{\frac{1}{2}} \leq C_{n, r}\left(H^{2}, H^{\infty}\right) \leq\left(\frac{2 n}{1-r}\right)^{\frac{1}{2}} .
$$

(b) D'autre part, le problème de calculer/estimer $c(\sigma, X, Y)$ peut être vu comme une interpolation intermédiaire entre celle dite de Carleson et l'interpolation individuelle de Nevanlinna-Pick.

(c) On trouve une autre motivation pour l'estimation de la constante $c(\sigma, X, Y)$ dans le calcul matriciel où on s'interesse à la norme du calcul fonctionnel: trouver $C>0$ optimale telle que si $A$ est une matrice $n \times n$ vérifiant $\sigma(A) \subset \sigma \subset \mathbb{D}$, telle que $\|A\|_{E \rightarrow E} \leq 1$ par rapport à une certaine norme sur $\mathbb{C}^{n}, E=\left(\mathbb{C}^{n},||.\right)$,

$$
\|f(A)\| \leq C\|f\|_{\infty},
$$

pour tout polynôme analytique $f$. Il est facile de voir que $C=c\left(\sigma, H^{\infty}, W_{a}\right)$, où $W_{a}$ est l'algèbre de Wiener des séries de Taylor absolument convergentes. Notons l'apparition d'un cas intéressant pour $f \in H^{\infty}$ telle que $f_{\mid \sigma}=\frac{1}{z \mid \sigma}$ (estimation du conditionnement et des normes d'inverses des matrices $n \times n$ ) ou telle que $f_{\mid \sigma}=\frac{1}{\lambda-z \mid \sigma}$ (pour l' estimation de la norme de la résolvante d'une matrice $n \times n$ ).

Un résultat de N. Nikolski (voir [13]) nous garanti que $c\left(\sigma, X, W_{a}\right)$ est majoré par 9n.c $\left(\sigma, X, H^{\infty}\right)$ pour tout espace de Banach $X$ de fonctions holomorphes dans $\mathbb{D}$ et que cette majoration est exacte ( $\operatorname{sur} X$ et $\sigma$, \# $\sigma \leq n$ ) à une constante numérique près.

\section{(3) Les espaces considérés}

(a) $X=H^{p}=H^{p}(\mathbb{D}), 1 \leq p \leq \infty$, les espaces de Hardy du disque $\mathbb{D}$,

(b) $X=l_{a}^{2}(\alpha):=l_{a}^{2}\left(\frac{1}{(k+1)^{\alpha-1}}\right), \alpha \geq 1$, l'espace à poids des fonctions $f(z)=\sum_{k \geq 0} \hat{f}(k) z^{k}$ vérifiant

$$
\sum_{k \geq 0}|\hat{f}(k)|^{2} \frac{1}{(k+1)^{2(\alpha-1)}}<\infty
$$

une description équivalente de cette même série d'espaces est:

$$
X=L_{a}^{2}(\beta):=L_{a}^{2}\left(\left(1-|z|^{2}\right)^{\beta} d x d y\right), \beta=2 \alpha-3>-1 \text {, l'espace de Bergman à poids des fonctions }
$$

holomorphes $f$ telles que

$$
\int_{\mathbb{D}}|f(z)|^{2}\left(1-|z|^{2}\right)^{\beta} d x d y<\infty .
$$

Pour $\beta=0$, on raccourcit la notation, $X=L_{a}^{2}$.

(c) On va un peu plus loin en considérant:

$X=l_{a}^{p}(\alpha):=l_{a}^{p}\left(\frac{1}{(k+1)^{\alpha-1}}\right), \alpha \geq 1,1 \leq p \leq \infty$, puis $X=L_{a}^{p}(\beta):=L_{a}^{p}\left(\left(1-|z|^{2}\right)^{\beta} d x d y\right), \beta>-1$, $1 \leq p \leq 2$. 


\section{B. Ce qui est montré}

Nous commençons par étudier le cas d'espaces de Banach généraux $X$ et $Y$ verifiant les propriétés naturelles suivantes:

$$
\text { pour tout } \epsilon>0, \operatorname{Hol}((1+\epsilon) \mathbb{D}) \text { est continument inclus dans } Y
$$

$$
\mathrm{Pol}_{+} \subset \mathrm{X} \text { et } \mathrm{Pol}_{+} \text {est dense dans } \mathrm{X},
$$

où $\mathrm{Pol}_{+}$désigne l'espace des polynômes analytiques à coefficients complexes $p, p(z)=\sum_{k=0}^{N} a_{k} z^{k}$,

$$
\begin{gathered}
{[f \in X] \Rightarrow\left[z^{n} f \in X, \forall n \geq 0 \text { and } \overline{\lim }\left\|z^{n} f\right\|^{\frac{1}{n}} \leq 1\right],} \\
{[f \in X, \lambda \in \mathbb{D}, \text { and } f(\lambda)=0] \Rightarrow\left[\frac{f}{z-\lambda} \in X\right] .}
\end{gathered}
$$

Lemme 0. Soient $X, Y$ deux espaces de Banach vérifiant les propriétés $\left(P_{i}\right), i=1 \ldots 4$. Pour tout $n \geq 1$, $r \in[0,1)$, on a $C_{n, r}(X, Y)<\infty$.

Puis, en étudiant le cas particulier où $Y=H^{\infty}$ et où $X$ parcourt les espaces décrits en A.(3)-(a) et A.(3)-(b), nous obtenons des majorations/minorations du type

$$
c_{1} \varphi_{X}\left(1-\frac{1-r}{n}\right) \leq C_{n, r}\left(X, H^{\infty}\right) \leq c_{2} \varphi_{X}\left(1-\frac{1-r}{n}\right),
$$

où $\varphi_{X}(t), 0 \leq t<1$ est la norme de la fonctionnelle d'évaluation $f \mapsto f(t)$ sur l'espace $X$.

Plus précisément, en ce qui concerne les espaces de Hardy $H^{p}$ du A.(3)-(a), on obtient le théorème suivant.

Théorème 1. Soit $p \in 2 \mathbb{Z}_{+}$. Il existe une constante $A_{p}$ dépendant de $p$ uniquement telle que pour tout $n \geq 1$, $r \in[0,1)$,

$$
\frac{1}{32^{\frac{1}{p}}}\left(\frac{n}{1-r}\right)^{\frac{1}{p}} \leq C_{n, r}\left(H^{p}, H^{\infty}\right) \leq A_{p}\left(\frac{n}{1-r}\right)^{\frac{1}{p}} .
$$

De plus, la majoration est vraie pour tout réel $p, 1 \leq p \leq+\infty$.

Quant au cas des espaces à poids (où de façon équivalente celui des espaces de Bergman à poids radial) du A.(3)-(b), $X=l_{a}^{2}(\alpha)=L_{a}^{2}(2 \alpha-3)$, on obtient l'encadrement suivant.

Théorème 2. Soit $\alpha \geq 1$ tel que $2 \alpha-1$ soit entier. Il existe des constantes a et A telles que pour tout $n \geq 1, r \in[0,1)$,

$$
a\left(\frac{n}{1-r}\right)^{\frac{2 \alpha-1}{2}} \leq C_{n, r}\left(l_{a}^{2}(\alpha), H^{\infty}\right) \leq A\left(\frac{n}{1-r}\right)^{\frac{2 \alpha-1}{2}},
$$

où les constantes $a$ et $A$ sont telles que $a \asymp \frac{1}{2^{3 N}(2 N) !}$ et $A \asymp N^{2 N}, N$ étant la partie entière de $\alpha$. De plus, la majoration est vraie pour tout réel $\alpha \geq 1$. (La notation $x \asymp y$ signifie qu'il existe des constantes numériques $c_{1}, c_{2}>0$ telles que $\left.c_{1} y \leq x \leq c_{2} y\right)$.

Enfin, en ce qui concerne le cas des espaces $X$ du A.(3)-(c), les résultats obtenus sont plus faibles et ne répondent pas, comme c'était le cas précédemment, à la conjecture faisant intervenir $\varphi_{X}$ définie ci-dessus. Nous donnons néanmoins des majorations/minorations pour la quantité $c\left(\sigma, X, H^{\infty}\right)$, parfois pour des $\sigma$ spécifiques et avec des écarts entre les bornes intervenant dans ces encadrements.

Théorème 3. (1) Soit $\alpha \geq 1$ et $X=l_{a}^{p}(\alpha)$. Pour $1 \leq p \leq+\infty$, il existe une minoration de $C_{n, r}\left(X, H^{\infty}\right)$ de l'ordre de $1 /(1-r)^{\alpha-1 / p}$. Pour $1 \leq p \leq 2$ (resp. $\left.2 \leq p \leq+\infty\right)$, il existe une majoration de $C_{n, r}\left(X, H^{\infty}\right)$ de l'ordre de $\left(\frac{n}{1-r}\right)^{\alpha-1 / 2}$ (resp. de l'ordre de $\left.\left(\frac{n}{1-r}\right)^{\alpha+1 / 2-2 / p}\right)$. 
(2) Soient $\lambda \in \mathbb{D}, \beta>-1,1 \leq p \leq 2$ et $X=L_{a}^{p}(\beta)$. Alors il existe une majoration de $c\left(\sigma_{\lambda, n}, X, H^{\infty}\right)$ de l'ordre de $\left(\frac{n}{1-|\lambda|}\right)^{(\beta+2) / p}$.

\section{Les moyens pour montrer cela}

\section{(a) Majorations}

(i) Nous avons choisi d'utiliser une interpolation linéaire

$$
T: f \mapsto \sum_{k=1}^{n}\left\langle f, e_{k}\right\rangle e_{k},
$$

où $\langle.,$.$\rangle est la forme sesquilinéaire de Cauchy \langle h, g\rangle=\sum_{k \geq 0} \hat{h}(k) \overline{\hat{g}(k)}$, et $\left(e_{k}\right)_{k=1}^{n}$ est la base de l'espace $K_{B}=$ $H^{2} \Theta B H^{2}$, dite de Malmquist, connue de façon explicite, (voir N. Nikolski, [12] p. 117)). Ce choix est justifié par le fait que si $X=H^{2}$, l'opérateur $T$ coïncide avec la projection orthogonale de $H^{2}$ sur $H^{2} \Theta B H^{2}$. Nous conserverons ce choix même dans le cas plus général où $X=H$ est un espace de Hilbert différent de $H^{2}$, car pour ce type d'espace la projection orthogonale de $H$ sur $H \Theta B H$ demeure implicite. D'autre part, si $X$ n'est pas un espace de Hilbert, trouver la "meilleure" interpolante de $f$ est encore moins clair, il y aura donc un prix à payer relativement à notre choix. A ce propos, en général la vraie interpolation optimale est non-linéaire, voir S.A. Vinogradov ([15]) pour les détails.

(ii) Pour le cas A.(3)-(a) (Théorème 1) avec $p=2$, on profite du fait que $e_{k} \in \mathcal{H}$ ol $\left(|z|<\frac{1}{r}\right)$ où $r=\max _{\lambda \in \sigma}|\lambda|$ pour majorer $\|g\|_{\infty}$. Pour généraliser au cas $p \geq 1$ quelconque on utilise un résultat d'interpolation de P. Jones notamment que $\left[H^{1}, H^{\infty}\right]_{\theta}=H^{p}$, voir [9].

Pour le cas A.(3)-(b) (Théorème 2), on utilise les points ci-après.

(iii) Pour $X=l_{a}^{2}(N+1)$, on fait apparaître la quantité $\left\|g^{(N)}\right\|_{H^{2}}$ (qui est comparable à $\|g\|_{X^{\star}}$ ) que l'on majore en fonction de $\|f\|_{H^{2}}$ à l'aide d'une inégalité type Bernstein sur les fonctions rationelles à pôles dans ${ }^{c} \overline{\mathbb{D}}$, que l'on montrera. Plus précisément, on démontrera le lemme suivant, qui est un analogue pour le disque d'un résultat de K. Dyakonov démontré dans le demi-plan, voir [7].

Lemme 1. Soit $g \in K_{B}=: H^{2} \Theta B H^{2}$. Alors

$$
\left\|g^{\prime}\right\|_{H^{2}} \leq \frac{5}{2} \frac{n}{1-r}\|g\|_{H^{2}}
$$

où comme toujours, $r=\max _{\lambda \in \sigma}|\lambda|$. Par récurrence,

$$
\left\|g^{(k)}\right\|_{H^{2}} \leq k !\left(\frac{5}{2}\right)^{k}\left(\frac{n}{1-r}\right)^{k}\|g\|_{H^{2}}
$$

pour tout $k=0,1, \ldots$.

Notre preuve est différente de celle de M. Dyakonov et elle donne en particulier une constante $(5 / 2)$ plus petite. En général, on notera que les inégalités type Bernstein ont déjà fait l'objet de nombreuses publications. Entre autres, le chapitre 7 du livre de P. Borwein et T. Erdélyi, voir [5], y est consacré. C'est aussi le cas de la thèse de A. Baranov, voir [1], et de l'ouvrage de R. A. DeVore and G. G. Lorentz, voir [6].

(iv) Enfin, comme en (ii), on interpole entre $l_{a}^{2}(N)$ et $l_{a}^{2}(N+1)$ (interpolation classique complexe entre espaces de Banach, voir [14] ou [4]).

(v) Pour traiter le cas A.(3)-(c), on fait de même qu'en (iv) mais entre $l_{a}^{p_{1}}(\alpha)$ et $l_{a}^{p_{2}}(\alpha)$, puis entre $L_{a}^{p_{1}}(\beta)$ et $L_{a}^{p_{2}}(\beta)$.

\section{(b) Minorations}

On se rend compte, grâce l'interpolation de Carleson, que la séquence la "pire" est probablement $\sigma=\sigma_{\lambda, n}=$ $\{\lambda, \ldots, \lambda\}$ ( $n$ fois). En effet, dans ce cas la constante de Carleson explose et, au travers de la majoration, (qui est vraie pour toute séquence $\sigma$ de $\mathbb{D}$ ),

$$
c\left(\sigma, X, H^{\infty}\right) \leq C_{I}(\sigma) \cdot \max _{1 \leq i \leq n}\left\|\varphi_{\lambda_{i}}\right\|
$$


où $\varphi_{\lambda}(f)=f(\lambda)$ et

$$
C_{I}(\sigma)=\sup _{\|a\|_{l} \leq 1} \inf \left(\|g\|_{\infty}: g \in H^{\infty}, g_{\mid \sigma}=a\right)
$$

est la constante de Carleson relative à $\sigma$, on comprend que pour $\sigma$ ayant une constante d'interpolation $C_{I}(\sigma)$ "raisonnable", la quantité $c\left(\sigma, X, H^{\infty}\right)$ se comporte comme $\max _{i}\left\|\varphi_{\lambda_{i}}\right\|$. En revanche, pour des sequences $\sigma$ "serrées", la constante $C_{I}(\sigma)$ peut être si grande que la dernière majoration peut en devenir très grossière .

(i) On remarque d'abord que

$$
c\left(\sigma_{0, n}, H^{2}, H^{\infty}\right) \geq \frac{1}{\left\|p_{n}\right\|_{H^{2}}}\left\|p_{n} \star K_{n}\right\|_{\infty} \geq \frac{1}{\sqrt{n-1}}\left(p_{n} \star K_{n}\right)(1) \geq \frac{1}{2} \sqrt{n},
$$

où $K_{n}$ désigne le noyau de Fejer d'ordre $n$, et $p_{n}=\sum_{k=0}^{n-1} z^{k}$.

(ii) Le cas $c\left(\sigma_{0, n}, X, H^{\infty}\right)$ pour $X$ espace de Hilbert du A.(3)-(b) se traite de la même façon, en remplaçant $p_{n}$ par une puissance de $p_{n}$.

(iii) La minoration de $c\left(\sigma_{\lambda, n}, H^{2}, H^{\infty}\right)$ se "déduit" de (ii) en considérant, au lieu de la fonction $p_{n}=\sum_{k=0}^{n-1} z^{k}$, une fonction construite à partir de $p_{n} \circ b_{\lambda}$, ou plus simplement $p_{n} \circ b_{r}, 0 \leq r<1$ puisque les normes considérées sont invariantes par rotation. On prouve ainsi la minoration du Théorème 1 pour $p=2$.

(iv) Encore une fois, le cas $c\left(\sigma_{\lambda, n}, X, H^{\infty}\right)$ pour $X$ espace de Hilbert du A.(3)-(b) se traite comme en (iii), en remplaçant $p_{n}$ par une puissance de $p_{n}$. L'observation principale réside dans le fait que $X=l_{a}^{2}(\alpha)=\varphi\left(H^{2}\right)=$ $H(\varphi \circ K)$ dans le sens d'Aronszajn-deBranges, (voir [12] p.320, point ( $k$ ) de l'Exercice 6.5.2), avec $\varphi(z)=z^{2 \alpha-1}$ et $K(\lambda, z)=k_{\lambda}(z)=\frac{1}{1-\lambda z}$. En particulier, on utilise l'inégalité suivante, vraie pour tout $f \in H^{2}$ :

$$
\|\varphi \circ f\|_{X}^{2} \leq \varphi\left(\|f\|_{2}^{2}\right)
$$

Il est bon de noter que l'opérateur de composition par $b_{\lambda}$ stabilise les espaces du A.(3)-(a) et du A.(3)-(b) mais qu'en revanche ce n'est pas le cas pour les espaces $l_{a}^{p}(\alpha)$ pour $p \neq 2$ ce qui rend le problème de minoration mais aussi de majoration plus difficile.

(v) Enfin, pour montrer la minoration du Théorème 3, on utilise simplement que

$$
C_{n, r}\left(X, H^{\infty}\right) \geq\left\|\psi_{r}\right\|_{X}
$$

où $\psi_{r}(f)=f(r)$. (Mais la minoration ainsi obtenue n'est pas optimale).

\section{Remerciements}

Je tiens à remercier chaleureusement le Professeur Nikolai Nikolski pour son aide inestimable.

\section{REFERENCES}

[1] A. Baranov, Inégalités de Bernstein dans les espaces modèles et applications, Thèse soutenue à l'université de Bordeaux 1, 2005.

[2] L. Baratchart, Rational and meromorphic approximation in Lp of the circle : system-theoretic motivations, critical points and error rates., Computational Methods and Function Theory, World Scientific Publish. Co, 11 (1999) 45-78.

[3] L. Baratchart, F. Wielonsky, Rational approximation problem in the real Hardy space $\mathrm{H}_{2}$ and Stieltjes integrals: a uniqueness theorem, Constr. Approx. 9 (1993), 1-21.

[4] J. Bergh , J. Löfström, Interpolation Spaces. An Introduction, Springer-Verlag, Berlin-Heidelberg-New York, 1976.

[5] P. Borwein and T. Erdélyi, Polynomials and Polynomial Inequalities, Springer, New York, 1995.

[6] R. A. DeVore and G. G. Lorentz, Constructive Approximation, Springer-Verlag, Berlin, 1993.

[7] K. Dyakonov, Differentiation in Star-Invariant Subspaces I. Boundedness and Compactness, J.Funct.Analysis, 192 (2002), 364-386.

[8] J. Garnett, Bounded Analytic Functions, Academic Press, New York, 1981.

[9] P. W. Jones, $L^{\infty}$ estimates for the $\bar{\partial}$ problem in the half plane, Acta Math. 150 (1983), 137-152.

[10] P. Koosis, Carleson's interpolation theorem deduced from a result of Pick, Complex analysis, operators, and related topics. In V. Havin, and N. Nikolski, editors, 151-162, Oper. Theory Adv. Appl., 113, Birkhäuser, Basel, 2000.

[11] N.Nikolski, Treatise on the shift operator, Springer-Verlag, Berlin etc., 1986.

[12] N.Nikolski, Operators, Function, and Systems: an easy reading, Vol.1. AMS, Providence, 2002.

[13] N.Nikolski, Condition Numbers of Large Matrices and Analytic Capacities, St. Petersburg Math. J., 17 (2006), 641-682.

[14] H. Triebel, Interpolation theory, functions spaces, differential operators, North-Holland Pub. Comp., Amsterdam, New York, Oxford, 1978.

[15] S. A. Vinogradov, Some remarks on free interpolation by bounded and slowly growing analytic functions, Zapiski Nauchn. Sem. Leningrad. Otdel. Mat. Inst. Steklov. (LOMI), 126 (1983), 35-46, (Russian); Engl. translation: J. of Math. Sci., 27-1, (1984), 2450-2458. 
Equipe d'Analyse et Géométrie,

Institut de Mathématiques de Bordeaux,

Université Bordeaux, 351 Cours de la Libération, 33405 Talence, France.

E-mail address: rzarouf@math.u-bordeaux1.fr 